\title{
Launch Vehicle Electrical Power System Rocket-ground Wireless Interface Prototype
}

\author{
Xianting Bi, Jingang Zhang, Xinglai Wang, Wei Zhu \\ Beijing Institute of Astronautical Systems Engineering, Fengtai Strict, Beijing, China \\ Zhangjg2009@126.com
}

\begin{abstract}
:
To realize unattended test for launch vehicle, we design and implement the electrical power system rocket-ground wireless interface prototype by means of a short distance high speed wireless communication and high power wireless power supply integration technology that is adapted to test and launch mode. This work gives the overall design and application scheme. The wireless laser power supply and wireless communication integration system consists of high power laser, transmit optical antenna, receive photoelectric conversion array and wireless laser communication equipment (transmit and receive). The transmission and conversion efficiencies of wireless power supply and communication are promoted by optimization design of transmit and receive device, the performance of $2 \mathrm{kw}$ power supply at $25 \mathrm{~m}$ with $500 \mathrm{Mbps}$ bidirectional wireless communication capability can be achieved.
\end{abstract}

Key Words: rocket-ground wireless interface, laser, unattended test, optimization

\section{Introduction}

Launch vehicle electrical power system rocket-ground interface is used for power supply, instruction and data interaction between on-vehicle electrical system and ground test and control system during testing and launching. The traditional connection-separation devices used in launch vehicle electrical power system rocket-ground interface are pull-off connector and separation connector, which are complicate to operate, have low connection reliability and cannot realize automatic connection, therefore are unable to meet the front end unattended test demand of new launch vehicle ${ }^{[1]-[5]}$. With the rapid development of high speed wireless communicate technology represented by laser ${ }^{[6],[7]}$, microwave communication and high power wireless power supply technology represented by electromagnetic coupling and laser, the rocket-ground interface of launch vehicle can be upgraded from wire connection to wireless connection. The electrical system adapts wireless interface to realize wireless power supply and wireless communication integration, consequently, the mechanical connection can be cancelled and the operation is greatly simplified. In abnormal situation, rocket-ground control and power supply can be rapidly recover and launch vehicle safety are remarkably improved.

This program aimed at unattended operation in test and launching phase of launch vehicle, breaking through short distance high speed wireless communication and high power wireless power supply integration technology adapted to launch vehicle test and launching mode, and finally realizing electrical system rocket-ground wireless. 


\section{Wireless rocket-ground interface description}

To realize wireless power supply from ground to rocket and bidirectional communication, every section of the launch vehicle is installed with a wireless rocket-ground interface, as shown in figure 1. Electrical system can be powered through this interface during ground test phase, meanwhile, the bidirectional wireless communication between ground and rocket downlinks the digital data of electrical system states information( single equipment built-in test information, sensor information, navigation and guidance information, sequence control information, power supply and distribution information and et. al) and uplinks ground instructions( power supply and distribution instruction, test procedure control instruction, fault injection instruction, data setting, software setting and et. al). 

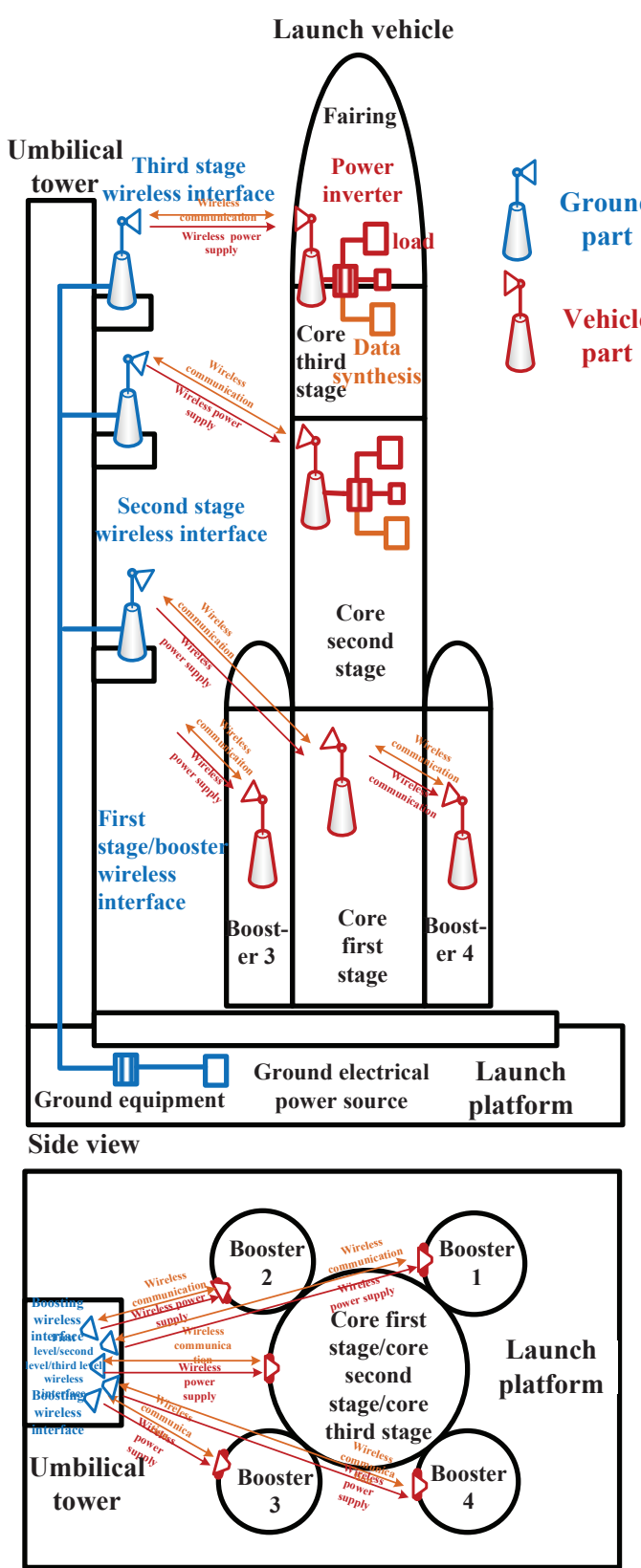

Vertical view

(a) boosting rocket-ground interface placed on umbilical tower

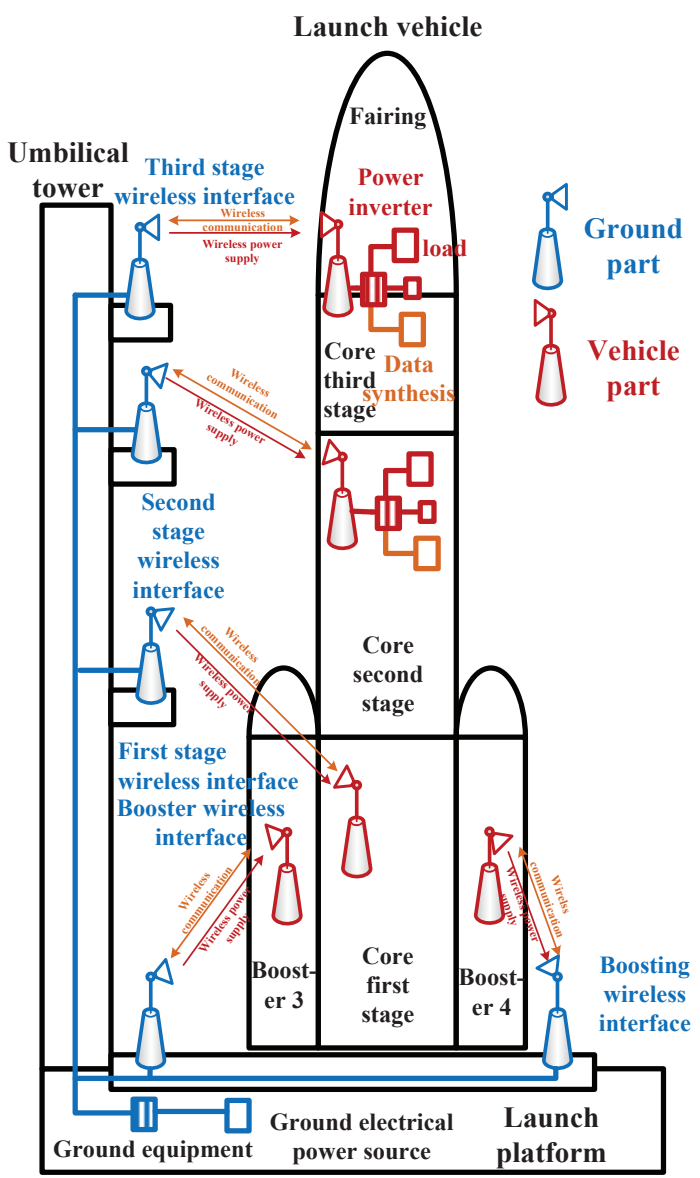

Side view

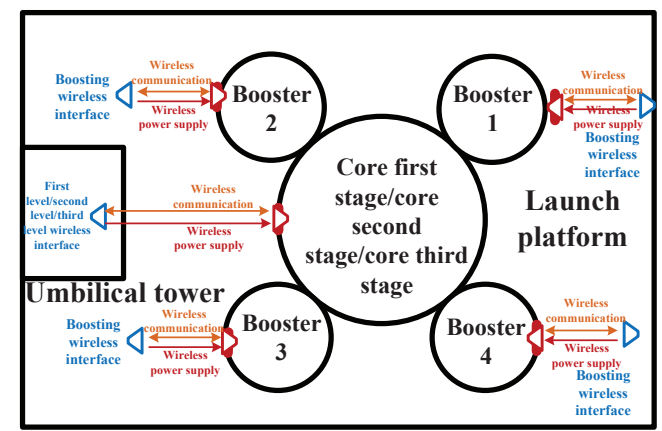

Vertical view

(b) boosting rocket-ground interface placed on launch platform

Fig. 1 wireless rocket-ground interface application

The ground part of booster stage wireless interface can be placed on umbilical tower or launch platform, and the onboard part can be placed on the outside of booster cabin wall, laser is employed for wireless power supply and rocket-ground communication. For the first, second and third stage, the ground parts are placed on the umbilical tower of launching platform, the onboard parts are placed on interstage or the outside of instrument cabin, the ground power supply and rocket-ground communication are also realized by laser. The ground part are connected to power source and communication equipment inside the launching platform with cable.

\section{Application Mode}

During ground testing, the rocket-ground 
wireless interface automatic connection, aided by optical aiming method to minimum the power loss of wireless power supply and communication, is firstly performed. The test instructions and data are transferred by wireless interface, and then distributed to related equipment. There are three application modes of wireless power supply:

(1) charge while using: in ground test, fill and prelaunch prepare phase, the relation between battery and wireless power supply is similar to mobile phone battery and charger. When onboard load is lower than wireless electric supply power, onboard equipment is powered by wireless power supply and the battery is simultaneously charged until full. when onboard load is higher than wireless electric supply power, both wireless power supply and battery provide energy to onboard equipment. The battery and wireless power supply are balanced by battery management system. (2) no charge while using: traditional analogy cable is replaced with rocket-ground wireless power supply, onboard battery is not used while ground testing, only wireless power supply provide energy to onboard equipment. (3) charge without using: to simplify up- and down-rocket operation, battery is used to provide energy during ground testing and charged with wireless

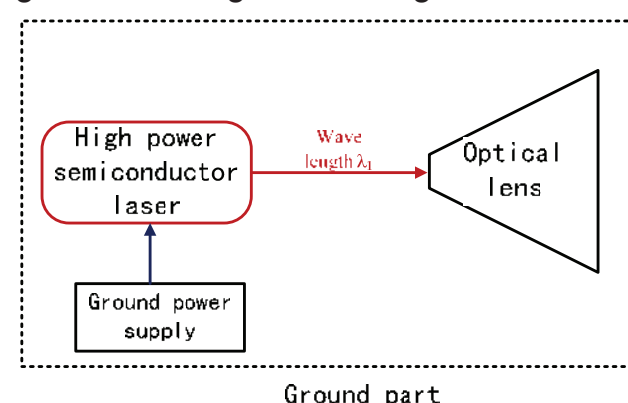

Fig. 2 laser wireless power supply basic structure
The ground power supply provide energy so that the high power laser can output high power laser. The cyber and transmit optical lens are coupled to output laser beam, which power supply during intermittency.

Before igniting, rocket-ground wireless power supply and communication are disconnected consequently, onboard equipment is then powered by onboard battery after power mode switching. If emergency cut-off happens after igniting, onboard equipment communication can be immediately recovered, ground can thus monitor and control of onboard equipment, onboard pyrotechnics short-circuit protection and key equipment power-off operation are performed according to the situation.

To get into the optimum work state, initial aiming is needed from ground to onboard receiver. Aiming error generated by rocket and ground sloshing and other external factor is compensated.

\section{Laser wireless power supply scheme}

Basic principle of laser wireless power supply scheme is shown in figure 2. It consists of two parts. The ground part, constructed with ground power supply, high power laser and optical lens, transmits high power wireless laser. The onboard part, constructed with photovoltaic convert array, power supply management equipment and load, is used to receive high power wireless laser signal.

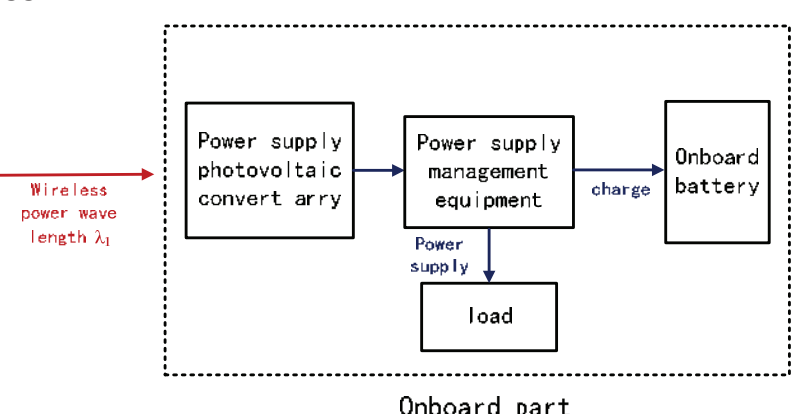

is then transmitted to onboard receiver through space chain after collimating of the optical system. To transform input optical energy to electrical power, the light spot 
received by onboard receiver is projected to the photovoltaic converter array constructed by laser photoelectric cell. Voltage transformation and filter are also needed.

To promote the received electrical power, both the transmitted power and the receive efficiency should be improved. For this reason, researches including multi-beam energy transmit, optical spot homogenization, multi-beam cooperate simulation and photovoltaic converter array optimization are conducted.

\section{Wireless power supply transmission efficiency optimization}

The overall electrical-optical-electrical transmission efficiency are as follows:

$$
\eta=P_{S} / \mathrm{P}_{R}=\eta_{1} \cdot \eta_{2} \cdot \eta_{3} \cdot \eta_{4} \cdot \eta_{5} \cdot \eta_{6}
$$

where $\eta 1$ represents laser electro-optical conversion efficiency; $\eta 2$ is antenna transmission efficiency; $\eta 3$ is laser transmission efficiency in space; $\eta 4$ is incident spot utilization efficiency; $\eta 5$ is pointing deviation efficiency; $\eta 6$ is optical-electrical conversion efficiency.

a) Laser optical-electrical conversion efficiency

The optical-electrical conversion efficiency of the present semiconductor laser are $35 \%$, which is the efficiency of common commercial lasers.

\section{b) Antenna transmission efficiency}

The transmit light path is constructed by a series of lens to shape, collimate and astigmatism correct the input beam. The transmittance of one lens surface can reach up to $99.9 \%$ with added reflection reducing coating.

This system includes transmission and reflection homogenization transmit antenna. Considering the homogenization effect and center spot energy area, the efficiency of the present transmission type homogenizer can reach up to $95 \%$, of reflection type can reach up to $97 \%$.

\section{c) Atmosphere scattering attenuation}

The experiment transmission distance is $25 \mathrm{~m}$, the atmosphere scattering attenuation is ca. $99.7 \%$ for $0.92 / \mathrm{km}$ ground atmosphere transmittance.

d) incident spot utilization efficiency

The key component of receiver is optical-electrical conversion unit, on which the photocell panel convert laser energy to electrical energy. The incident spot utilization efficiency is affected by two main factors: the battery arrangement duty ratio and the area ratio of photocell panel to incident spot.

To increase energy coverage, small photocell package are chosen in this design. The photocell slice is $10 * 10 \mathrm{~mm}$, the electric conduction electrode is $1 \mathrm{~mm}$, the gap between adjacent photocell is $0.5 \mathrm{~mm}$, therefore the actual area of every photocell $10.5 \mathrm{~mm} * 12.5 \mathrm{~mm}(132.25 \mathrm{~mm} 2)$ and the irradiation efficiency of single slice is $76.19 \%$. Array lens package can effectively increase the coverage efficiency to $91.4 \%$.

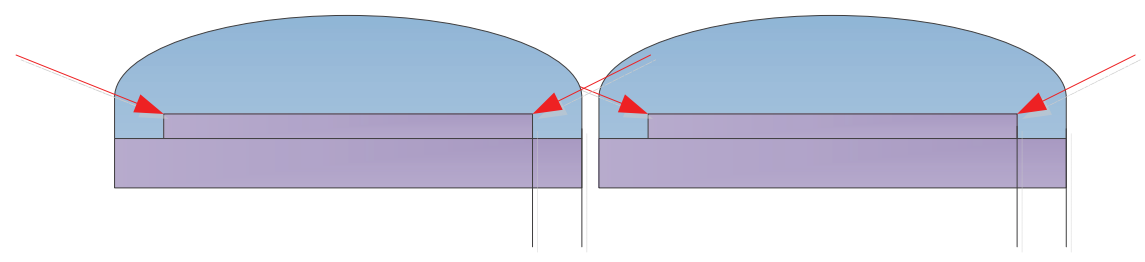

Fig. 3 photocell slice package

For a $400 \mathrm{~mm}$ diameter target surface, the photocell distribution efficiency is $92 \%$ 
without considering laser communication antenna, $90 \%$ considering laser communication antenna.

e) photo-electrical conversion efficiency

By measuring the open circuit voltage and short circuit currency of GaAs battery, the conversion efficiency is acquired as $40 \%$.

\section{f) Aiming adaption error}

The light spot coverage efficiency will decrease when the incident energy beam deviation happens. For the present stepper motor turntable, a $2 \mathrm{~cm}$ deviation at $25 \mathrm{~m}$ will decrease coverage efficiency to $97.5 \%$.

\section{g) Transmission efficiency synthesize}

According to the above analysis, the total electrical-electrical conversion efficiency is $13.4 \%$, the total optical-electrical conversion efficiency is $33.59 \%$. The data is shown in table 1.

Tab. 1: Transmission efficiency

\begin{tabular}{|l|c|c|}
\hline Num. & item & efficiency \\
\hline 1 & $\begin{array}{c}\text { Laser } \\
\text { optical-electrical } \\
\text { conversion efficiency }\end{array}$ & $40 \%$ \\
\hline 2 & $\begin{array}{c}\text { Optical transmission } \\
\text { antenna efficiency }\end{array}$ & $\begin{array}{c}95 \% \text { or } \\
97 \% \text { avg. } \\
96 \%)\end{array}$ \\
\hline 3 & $\begin{array}{c}\text { Atmosphere } \\
\text { scattering attenuation } \\
\text { efficiency }\end{array}$ & $99.7 \%$ \\
\hline 4 & $\begin{array}{c}\text { Incident spot } \\
\text { utilization efficiency } \\
\text { Pointing deviation } \\
\text { efficiency }\end{array}$ & $97.5 \%$ \\
\hline 5 & $\begin{array}{c}\text { Photoelectrical panel } \\
\text { optical-electrical } \\
\text { conversion efficiency }\end{array}$ & $45 \%$ \\
\hline
\end{tabular}

\section{Conclusion}

This program provides an integrated design of short distance high speed wireless communication and high power wireless power supply, thus realizes electrical system rocket-ground wireless interface. On this basis, future launch vehicle rocket-ground connection can be more flexible and be further simplified, which is important for unattended test and rapid test.

\section{Reference}

[1] Z. S. Liu, Z. Zhang. LM-2F Manned Launch Vehicle. Missiles and space vehicles, 2004, 1(267): 6-12.

[2] C. Q. Du, J. L. Pan. Integrated Design of Ground Test Launch and Control System for New Generation Launch Vehicle. Aerospace Control, 2004, 22,2:50-52.

[3] D. Li, J. Wang, W. Hei, et al. The General Scheme and Key Technologies of CZ-5 Launch Vehicle. Misiles and space vehicles, 2017, 353(3): 1-5.

[4] Y. LU. Space Launch Vehicle's Development in China. Astronautical Systems Engineering Technology, 2017, 1(3): 1-8.

[5] Z. Y. Wang, H. P. Chen, Y. Q. Zhu, et al. Survey and Review on Rapid Test Technology of Launch Vehicle. Journal of North University of China(natural science edition), 2017, 38(3): 307-315.

[6] Spaceward Foundation. Power Beaming(climber) Competition[EB]. http://www.spaceward.org/elevator2010-pb(a ccessed December 08).

[7] V. Khvostikov, S. Sorokina, N. Potapovich, et al. AIGaAs Converters and Arrays for Laser Power Beaming. International Conference on Concentrator Photovoltaic Systems, 2015, 1679(1): 187-194. 